\title{
A Survey Study on the Needs of Left-behind Children in China
}

\author{
Fei Xie \\ School of Marxism \\ Wuhan University of Technology \\ Wuhan, P. R. China \\ whutxf1992@sina.com
}

\begin{abstract}
This paper is to study the life situation and demand of the so-called left-behind children based on a questionnaire survey of 401 respondents in Hubei province, China. The result indicated that most of the left-behind children had no tutoring help in study, received normal level of care from their guardians, had low frequency of communication with their parents, received not enough psychological counseling at school, and perceived a little support from the government or social organizations. Left-behind children demanded academic counseling, family education and emotional care, and external support. Finally, we propose policy implications to meet the needs of left-behind children at levels of family, government and social organizations, and to better promote the physical and mental health of left-behind children.
\end{abstract}

Keywords_left-behind children; needs; life situation; China

\section{INTRODUCTION}

Since the Reform and Openness of China, the demand of labor force in the Chinese market increased dramatically. A large amount of surplus rural labors gathered in cities and towns. However, due to the dual structure in urban and rural areas, the migrant people find that it is quite hard to move their whole families to where they work and must leave their underage children in their hometown to be taken care of by others, resulting in the large number of left-behind children. As of now, the number of left-behind children in rural China has reached 9 million and 20 thousand [1]. Most of the left-behind children have stayed away from their parents for more than one year, unable to talk to their parents regularly to obtain emotional care and psychological comfort [2]. They also need academic support after school, but their guardians are mostly their grandparents. The guardians hold outdated thinking and ideas, having limited energy due to their physical and mental health conditions, along with low scientific and cultural quality, thus they could not take the responsibility of academic tutoring [3]. Compared with other children, left-behind children have more serious problems due to missing of family love and psychological comfort and lack of security and safety. Based on a survey investigation, this paper aims to understand the needs of Chinese left-behind children in areas of academic tutoring, family education, emotional care and social support, and put forward suggestions on how to improve the care of left-behind children.

\section{RESEARCH METHODS}

The survey was conducted in Huangzhou District, Huanggang City, Hubei Province located in central China. Based on the list of left-behind children provided by the local Department of Civil Affairs, the research team conducted a household survey in 35 villages and 11 communities from June to September 2017, screened and revised the list of left-behind children and interviewed each of them, completed the questionnaires of 401 left-behind Children. The survey included validation of the basic information of left-behind children and their guardians, the status quo of academic counseling, family education and emotional relationships, external social support to understand the needs of left-behind children.

\section{RESULTS}

\section{A. Information of the respondents and their guardians}

Among the 401 respondents, left-behind girls and boys accounted for $45.25 \%$ and $54.75 \%$ of the total sample respectively. Children aged 6 years and under and children aged $7-12$ years accounted for $37.76 \%$ and $46.43 \%$ of the total sample, separately. Most left-behind children were in the elementary and pre-primary school stage, accounting for $34.74 \%$ and $46.84 \%$ of the total sample respectively.

Left-behind children with both parents out of town working accounted for $81.80 \%$ of the total sample. $98.75 \%$ of the left-behind children were taken care by their grandparents. The grandparents who had no education or only elementary school education accounted for $37.09 \%$ and $34.09 \%$ respectively of the total samples. Their cultural knowledge level was very low. Meanwhile most grandparents suffered from diseases such as diabetes and hypertension, and in poor health conditions.

\section{B. Needs of academic counseling}

In this study, left-behind children's academic performance was at medium level. 35.86\% of them had a "good" academic records and $40.58 \%$ of the total sample had "normal" academic records. Most left-behind children needed academic counseling, but the majority of them did not have access to the counseling at all. Those who have comprehensive academic counseling accounted for only $17.54 \%$ of the total sample. 
The guardians who could only take care of the daily lives of left-behind children, or be responsible for personal safety accounted for $98.00 \%$ and $60.10 \%$ of the total sample respectively. They were not able to provide ideological and moral education and supervise and mentor the left-behind children with their study after school. However, since left-behind children were in the critical stage of their studies, they had a huge demand for academic mentoring. At the same time, ideological and moral education was mainly carried out in school, not a family priority. The juvenile's ideological development was immature, so they needed positive guidance.

\section{Needs of family education and emotional care}

Most of the left-behind children had certain level of communication with their guardians. 38.19\% of the total sample had the normal communication frequency. Generally left-behind children perceived their guardian's education style at normal level. $78.68 \%$ of the total samples rated their guardian education style as normal. $9.14 \%$ of the total sample evaluated it "relatively poor, need improvement" while $1.27 \%$ gave the rating as "completely unacceptable". Rating of "very good" only accounted for $10.91 \%$ of the total sample.

Grandparents as guardian was the most common style for the left-behind children. Grandparent generation usually had old values, more rigid mode of thinking model, and had a generation gap with left-behind children. Left-behind children tended to have stronger rebellious psychology. Grandparents often rebuked and reprimanded the left-behind children for their mistakes and misbehaviors which may cause children to hide mistakes and give up on themselves easily, or grandparents may neglect kids' bad behaviors resulting in poor self-discipline and other problems.

Most parents could not come home frequently due to the travel cost and not much free time due to their working in service business. The survey showed that parents coming home at one to two years intervals, accounting for $51.25 \%$ of the total sample. Left-behind children had few opportunities to meet their parents. $92.10 \%$ of the total sample communicated with their parents by phone. It was still not common to use Internet and other modern contact methods which only accounted for $26.69 \%$ of the total sample. Emotional contact method was still very unimodal, and it was difficult to observe the facial expression and gestures of the people on the other side of the phone, unable to meet the emotional needs of left-behind children care.

Overall the frequency of communication between left-behind children and their parents was at a low level. Only $35.19 \%$ of the total samples were rated as high frequency of communication. Left-behind children and their parents remain separated for long period of time. Parents did not fully understand their children's hobbies, and living habits. Left-behind children starved for parental care, but on the other hand, when they got together, they felt strange and not communicate well, easy to produce mood swings.

\section{Needs of external support}

The left-behind children who were strongly or generally willing to have a chat with their teachers and friends accounted for $28.46 \%$ and $54.83 \%$ of the total sample. $79.37 \%$ and $12.01 \%$ of the total left-behind children believed that there were few or no psychological counseling activities in schools. They may talk with their friends or teachers but only scratch the surface. Left-behind children could not be effectively relieved of their emotional and psychological stress. Psychological counseling and relief were needed to overcome their negative emotions such as low self-esteem and irritability to enhance the emotional control ability of left-behind children.

Left-behind children who perceived "no" support or "do not know" about any support from government or social organization rated $49.37 \%$ and $15.87 \%$ of the total samples respectively. It was at a lower frequency that guardians took the initiative to seek support from government or social organizations, only $3.75 \%$ of the total samples often asked for voluntary help from the government or social organizations while $70.50 \%$ of the total sample had never sought help from the government or social organizations.

\section{COUNTERMEASURES AND SUGGESTIONS}

\section{A. Family level}

Yu et al (2013) found that smooth communication with parents is the primary factor affecting the life events of left-behind children in rural areas [4]. More parent-child communication is beneficial to reduce the negative impact on left-behind children [5]. Thus parents of left-behind children should be more aware of the importance of parent-child communication and increase the number of return visits to reduce the waiting cycle time for children and try to enrich the communicating content and form. Zhang (2012) proposed that the communication between left-behind children and their parents over the telephone cannot achieve good emotional communication and recommended the usage of modern Internet communication methods [6]. Dai (2012) recommended to use letters to enhance parent-child relationship [7]. Parents should enrich their communication methods and channels, combine modern Internet media and traditional letter-writing methods. Using modern communication tools such as WeChat and QQ Video etc. to enable the communication of body languages, facial expressions and emotions. Those methods are much faster and more convenient and enhance the effectiveness of emotional communication. Correspondence with letters is good for expressing emotions and has the advantages of saving for reading at any time. Parents can take advantage of their spare time to write paper letters and e-mails to enhance their communication with their left-behind children.

Whether the guardians have good education quality and scientific education methods and education concepts mostly determines the quality of education of the grand parenting and affects the healthy growth of left-behind children [8]. Grandparent guardians should change their understanding of education mode, pay more attention to the guidance of left-behind children, especially the ideological and moral education and mental health of left-behind children, enhance the comprehensive training of left-behind children, rather than focus on the simple evaluation standard with Academic achievement at school. 


\section{B. Government level}

The government should promulgate regulations on protection of fundamental rights and interests for migrant workers such as working hours, environment and remuneration, and provide protection and basic public service for migrant workers to care for their children, continue to improve and implement the "return home employment" policy, vigorously develop economy in townships so that parents of left-behind children can find jobs in their hometown. Economic policies should be more lenient to the provinces that have larger number of migrant workers. Reduce the distance between home and working locations of those migrant workers, guarantee of the local school enrollment rights of left-behind children, cancel any disguised off-site admission thresholds to achieve a real educational equity, weaken the education and medical barriers associated with the household registration system to reduce the living and educational cost. All measures mentioned above will help to reduce the number of left-behind children.

Government departments should implement the policy of helping left-behind children, enrich the contents and forms of policy advocacy, and communicate the policy of helping left-behind children through the multiple agencies including autonomous agencies, schools and social organizations in urban and rural areas (villagers' committees), and establish online special platform for left-behind children care and protection, clarify the left-behind children and their guardians who seeking help, expand their channels for seeking help, set up a help-seeking system for left-behind children with various agencies include government, social organizations and schools, standardize help-seeking procedures and assistance methods etc.

\section{Social organizations level}

Social organizations should innovate in the form of services and work in left-behind children's living and learning locations. Based on the demands in multi-level emotional care, academic support and the improvement of the quality of guardians, innovate new forms of activities that fit the characteristics of children's grown period and unfold diverse and comprehensive professional services.

Launch parental education. By way of family interviews and other means to understand and assess the left-behind children's psychological, behavioral conditions and their family situation, regularly organize training and seminar on topics like caretaker parenting and knowledge and culture training and learning, promote the correct idea of parenting education, education and communication skills. At the same time, strengthen ties with parents of left-behind children to keep them updated with the status of their children, assist and strengthen their emotional ties, provide guidance of communication skills between parents and left-behind children. Remove any cultural level and technical capability limitation in the communication process.

Open 4:30 classrooms to provide after school tutoring for left-behind children. Start group activities with themes in interpersonal relationships, teacher-student relationship, parent-child relationship etc., pay close attention to spiritual and cultural needs of left-behind children, schedule regular cultural activities in calligraphy, dancing, film watching etc. and organize calligraphy, reading, basketball and other interest groups and training.

\section{SUMMARY}

The study found that more than half the parents of left-behind children came home once in one or two years. The communication frequency between left-behind children and their parents were relatively low. Telephone was the most common way of communication between parents and their left-behind children while nobody communicates by letters. Most guardians of left-behind children were from grandparent generation. Since the guardians of left-behind children were mostly from grandparent generation, usually of older age and less educated, it became more difficult to provide tutoring and guidance to their grandchildren. The overall evaluation of their education style from left-behind children was average or poor.

The survey data also indicated that left-behind children and guardians did not have enough understanding of the assistance policies from government or social organizations, there was a lack of channels for seeking help and guardians' initiative to seek help was also low. There were room for improvement for government or social organizations in their efforts to help left-behind children and propaganda.

In conclusion, the left-behind children demanded academic counseling, family education and emotional care, and external support. For better promoting the physical and mental health of left-behind children, further effort are supposed to be devoted on enhancing parent-child relationship, providing grand parenting education training, providing more policy support from the government and professional support from the social organizations.

\section{REFERENCES}

[1] Ministry of Civil Affairs, (2016). Bulletin about investigation of the basic situation of left-behind children in rural areas and description of "Combined guardianship, grow up together" special campaign in care and protection of left-behind children.

[2] M. Wen and D. Lin (2012). Child development in rural china: left-behind children by their migrant parents and children of nonmigrant families. Child Development, 83(1), 120-136.

[3] Y. Bai, L. Zhang, C. Liu, Y. Shi, D. Mo, and S. Rozelle (2016).Effect of parental migration on the academic performance of left-behind children in north western china. (1), 1-17.

[4] Y. Yu, Y. Ao, H. Shi, and Y. Zhu (2013).Various Life events and family impact factors with left-behind children. Chinese Public Health, 29 (03): 339-342(in Chinese)

[5] Q. Yang, L. Zhou, Y. Hu, C. Zhu, and H. Sun (2014). Effects of parent-child communication on behavioral problems of left-behind children in rural areas: the regulatory role of hope. Chinese Journal of Clinical Psychology, 22 (06): 1118- 1120. (in Chinese)

[6] S. Zhang, D. Huang, X. Liu, F. Yang, M. Jiang, Q. Lan, W.Yuan, Y. Zhao (2012). Investigation of parent-child relationship of left-behind children in a rural county in Sichuan province. Modern medicine and health, 28 (06): 829-831. (in Chinese)

[7] B. Dai (2012). Psychological characteristics of left-behind children in rural areas and educational strategies. Education Theory and Practice, 32(13):44-47. (in Chinese) 
[8] Z. Li, X. Zou, P. Zhu (2013). Empirical research on the competency of grandparent generation guardian of left-behind children in rural areas. Chinese Journal of Health Psychology, 21 (03): 422-42. (in Chinese)

[9] Decision of the State Council on Speeding up the Development of Modern Vocational Education,2014-05-29.

[10] Y ZHU. The Types and Operating Mechanism of School - Enterprise Cooperation in Higher Vocational Colleges J. Vocational and Technical Education,vol.35, pp.55-57, 2015. 